Bio - grafía. Escritos sobre la Biología y su Enseñanza. ISSN 2027-1034

Edición Extraordinaria. p.p. 1415-1422

Memorias del IX Encuentro Nacional de Experiencias en Enseñanza de la Biología y la

Educación Ambiental. IV Congreso Nacional de Investigación en Enseñanza de la Biología.

\title{
EL DESARROLLO DE LA OBSERVACIÓN Y LA INDAGACIÓN EN ESTUDIANTES DE QUINTO GRADO DE LA IE JUAN JACOBO ARAGÓN DE FONSECA - LA GUAJIRA
}

\author{
THE DEVELOPMENT OF OBSERVATION AND INQUIRY IN FIFTH STUDENTS OF IE \\ JUAN JACOBO ARAGÓN DE FONSECA - LA GUAJIRA
}

\section{Betty Ruth Blanchar Pérez ${ }^{1}$ Carlos Humberto Barreto Tovar ${ }^{2}$}

\section{RESUMEN}

Una de las dificultades presentadas en los estudiantes quinto grado es la debilidad en las competencias que atañen al pensamiento científico; muestra de ello son los resultados de las pruebas Saber. En esta investigación se logró caracterizar en las clases de tecnología e informática los niveles de observación planteados por Santelices (1989); formulación de preguntas e indagación por (Furman \& García, 2014) de objetos o del entorno del aula. Se logró a evidenciar que los estudiantes se encontraban en su gran mayoría ubicados en el nivel $1 \mathrm{~A}$ que corresponde a identificar y denominar formas básicas y colores en objetos diversos, y muchos otros sin categorizar. Por otra parte, en cuanto a la indagación se demuestra que la tipología de preguntas planteadas por los niños se ubican en el primer nivel según Santelices (1989), que corresponden a preguntas para obtener un dato o concepto, sobresaliendo las preguntas atípicas.

Luego, mediante el trabajo de clase enmarcado en la Enseñanza para la Comprensión (EpC), utilizando como estrategias las rutinas de pensamiento, el resultado ha mejorado. Se ha logrado evidenciar que los niveles de observación han aumentado, ya que al principio de este ciclo solo observaban la constitución general del objeto, ahora detallan características más concretas del mismo. En cuanto a las preguntas, los estudiantes se plantean de segundo nivel, preguntas que conllevan a la comprensión.

PALABRAS CLAVE: Observación, indagación, desarrollo del pensamiento científico, competencias científicas, Enseñanza para la Comprensión.

\begin{abstract}
One of the difficulties presented in the fifth grade students is the weakness in the competences that concern scientific thought; proof of this are the results of the Saber tests. In this research it was possible to characterize in the classes of technology and informatics the levels of observation proposed by Santelices (1989); formulation of questions and inquiry by (Furman \& García, 2014) objects or classroom environment. It was demonstrated that the students were mostly located at level $1 \mathrm{~A}$, which corresponds to identifying and naming basic

\footnotetext{
${ }^{1}$ Universidad de La Sabana. Facultad de Educación. Maestría en Pedagogía Ext. La Guajira. Correo: bettyblpe@unisabana.edu.co

2 Universidad de La Sabana. Facultad de Educación. Maestría en Pedagogía Ext. La Guajira. Correo: carlos.barreto2@unisabana.edu.co
} 


\section{Bio - grafía. Escritos sobre la Biología y su Enseñanza. ISSN 2027-1034}

Edición Extraordinaria. p.p. 1415-1422

Memorias del IX Encuentro Nacional de Experiencias en Enseñanza de la Biología y la Educación Ambiental. IV Congreso Nacional de Investigación en Enseñanza de la Biología.

shapes and colors in various objects, and many others without categorizing. On the other hand, as far as the inquiry is concerned, it is shown that the typology of questions posed by children are located at the first level according to Santelices (1989), which correspond to questions to obtain a data or concept, outstanding atypical questions.

Then, through the class work framed in the Teaching for Understanding (EpC), using as strategies the thought routines, the result has improved. It has been shown that the levels of observation have increased, since at the beginning of this cycle they only observed the general constitution of the object, now they detail more specific characteristics of the same. As for the questions, students are asked about second level questions that lead to understanding.

KEY WORDS: Observation, inquiry, development of scientific thinking, scientific competences, Teaching for Understanding.

\section{INTRODUCCIÓN}

El desarrollo del pensamiento científico se encuentra enmarcado en unas competencias que pretende "hacer más pertinente la ciencia para la vida cotidiana de los estudiantes" (Meinardi, E. 2010). En ese sentido, la Organización de las Naciones Unidas para la Educación, Ciencia y Cultura (citado en las Orientaciones Generales para la Educación en Tecnología, 2008) plantea "a la alfabetización en tecnología como un propósito inaplazable de la educación porque con ella se busca que los individuos y grupos estén en capacidad de comprender, evaluar, usar y transformar objetos, procesos y sistemas tecnológicos, como requisito para su desempeño en la vida social y productiva

Las prácticas pedagógicas que implementa el docente en el aula repercuten significativamente en el aprendizaje de los estudiantes; de allí que como docente se debe reflexionar sobre su quehacer dentro de la enseñanza y por lo tanto comenzar a hacer ciclos de reflexión sobre su praxis; de esta manera el docente entraría a darse cuenta si lo que hace se encuentra en sintonía con lo que pretende conseguir en sus estudiantes. El desarrollo de actitudes científicas y tecnológicas tiene que ver con las habilidades que son necesarias para enfrentarse a un ambiente que cambia rápidamente y que son útiles para resolver problemas, proponer soluciones y tomar decisiones sobre la vida diaria.

\section{Contexto}

La institución Educativa Juan Jacobo Aragón se encuentra ubicada en el perímetro urbano del municipio de Fonseca en el Departamento de La Guajira; ahora recientemente nombrada zona veredal debido a las negociaciones de paz que existen entre el gobierno nacional y los militantes de la guerrilla de las FARC; puesto que existe un campamento en la zona rural de este municipio. La Institución cuenta con 1.900 estudiantes aproximadamente repartidos en sus dos sedes, la sede de primaria ubicada en la principal cuenta aproximadamente con 600 estudiantes los cuales pertenecen a los estratos socioeconómicos 1 y 2 en su gran mayoría con padres que laboran en la informalidad, el grado quinto $\mathrm{A}$ en la cual he desarrollado esta investigación cuenta con 30 estudiantes que oscilan entre los 9 a 11 años de edad; estos niños son provenientes de los barrios subnormales del municipio, en la que son carentes las condiciones básicas para desarrollar actividades lúdicas. 
Bio - grafía. Escritos sobre la Biología y su Enseñanza. ISSN 2027-1034

Edición Extraordinaria. p.p. 1415-1422

Memorias del IX Encuentro Nacional de Experiencias en Enseñanza de la Biología y la Educación Ambiental. IV Congreso Nacional de Investigación en Enseñanza de la Biología.

\section{Problemática}

A partir de los resultados de las Pruebas Saber 2015, en los grados quintos en donde se evidenció la debilidad en la competencia científica de indagación. En este sentido, se planificó el desarrollo de una actividad diagnóstico para elaborar un registro de los niveles de observación e indagación de los estudiantes del grado Quinto A de la institución Educativa Juan Jacobo Aragón.

En este orden de ideas, se les sugiere a los estudiantes realizar una observación de su entorno, donde deberían tomar apuntes sobre todo lo observado para después exponer sus observaciones en clases. En un segundo momento fueron llevados a la sala de tecnología e informática para realizar observación a una maqueta correspondiente a un ecosistema; la docente les explicó dicha actividad, haciéndole énfasis en lo que era ver y observar; sugiriéndoles que le prestaran mucha atención a los detalles de las mismas; colores, formas, tamaños, olores, texturas y que deberían tomar nota de todo lo observado (Ver gráfica 1).

Resultados actividad de Observación Grado Quinto

\section{NIVELES DE OBSERVACIÓN}

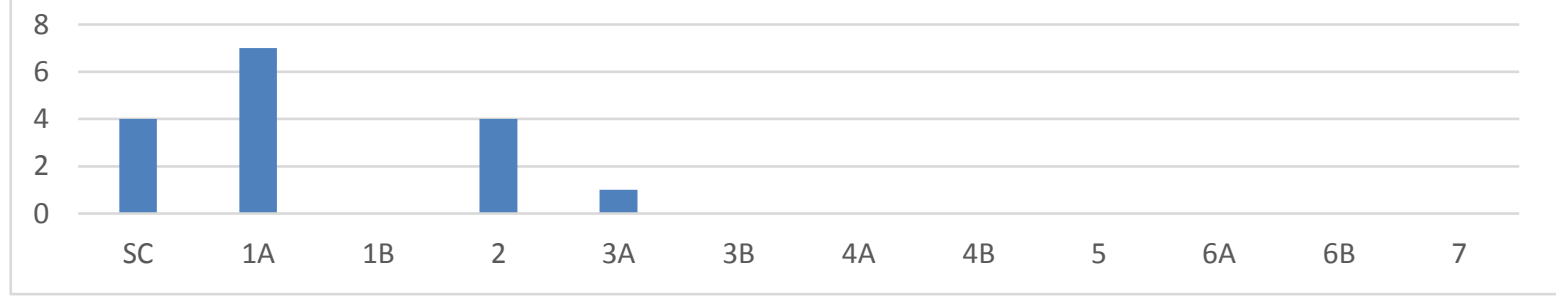

Gráfica 1. Observacion de una maqueta correspondiente a un ecosistema.

Seguidamente se les pidió realizar preguntas de todo lo observado, aquí la docente le comenta que las preguntas deben ser propuestas con las palabras que se usan para este fin. Esta información junto con las anotaciones de la observación fue entregada por cada estudiante para tabular la información (ver gráfica 2).

Resultados actividad de Indagación de Grado Quinto:

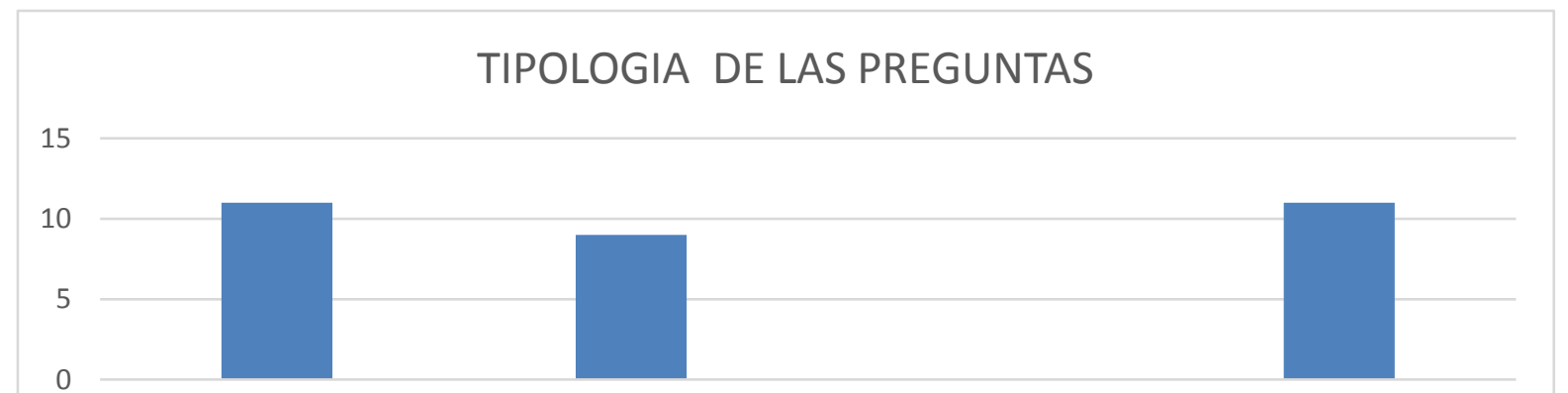

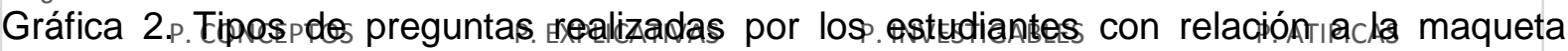
observada. 
Bio - grafía. Escritos sobre la Biología y su Enseñanza. ISSN 2027-1034

Edición Extraordinaria. p.p. 1415-1422

Memorias del IX Encuentro Nacional de Experiencias en Enseñanza de la Biología y la Educación Ambiental. IV Congreso Nacional de Investigación en Enseñanza de la Biología.

Luego de obtener los resultados de los tipos de preguntas realizadas por los estudiantes, la docente entra a categorizarlas, para determinar en qué nivel de indagación se encuentra cada uno de ellos (Ver gráfica 3 ).

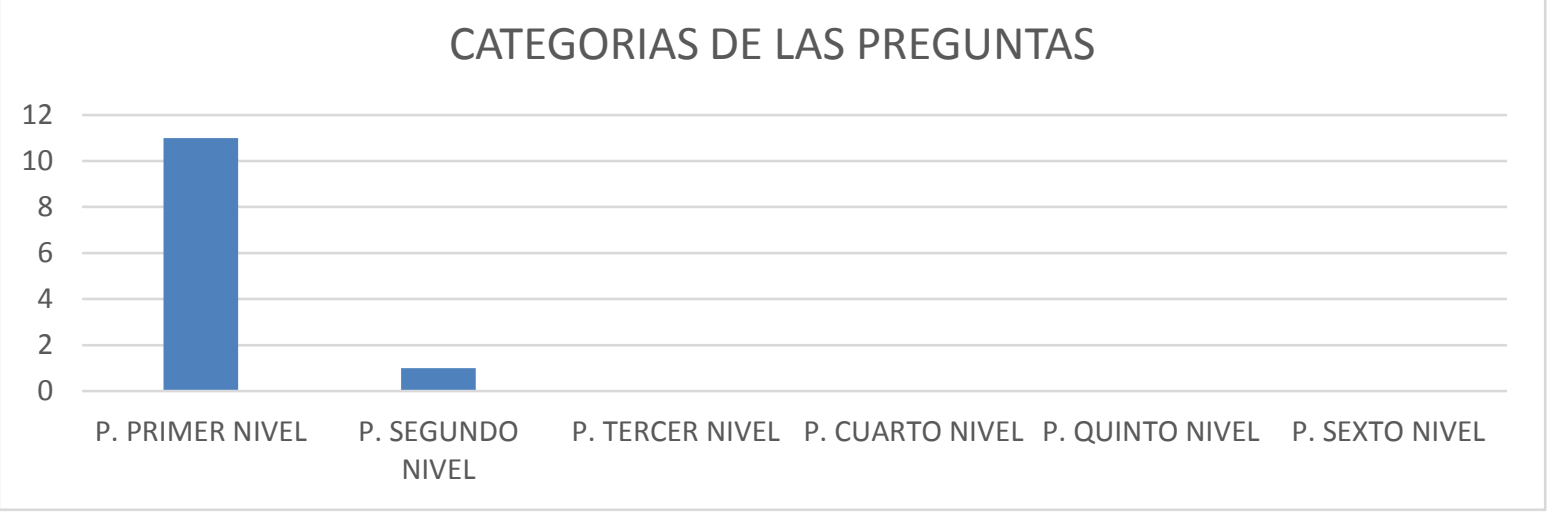

Gráfica 3. Categorías de preguntas en relación con la observación de la maqueta de los estudiantes de quinto.

Es evidente la problemática sobre el pensamiento científico-tecnológico y por ende en las competencias del uso del conocimiento científico, observación e indagación. Para la cual el docente debe formular estrategias que permitan el fortalecimiento de estas desde su quehacer en el aula de clases.

Por lo tanto atendiendo a esta problemática, es conveniente considerar que las estrategias son: "una secuencia especifica de actividades, una forma particular de intervención del docente" según (Feldman, 2010). En este orden de ideas el docente debe reflexionar sobre cómo estaba desarrollando pensamiento científico en los estudiantes y por consiguiente las competencias que les permitan implementar este pensamiento en su entorno. Teniendo en cuenta lo anterior, se plantearon estrategias como las rutinas de pensamientos para realizar observación e indagación como medio para fortalecer las competencias científicas y el desarrollo de este pensamiento en los estudiantes, definiendo estas como: "Procedimientos, procesos o patrones de acción que se usan de manera repetitiva para manejar y facilitar el logro de metas o tareas especificas" (Richhart, Church \& Morrinson, 2014).

\section{METODOLOGÍA}

Para esta investigación se utilizó el enfoque cualitativo, descriptivo pues la problemática es una realidad del aula de clases que permite al docente extraer información de la misma, para luego ser interpretada; Por otra parte, se basa en la investigación acción participación, que le permite al docente reflexionar sobre sus problemáticas de aula, intervenir la situación, analizar los resultados y reflexionar sobre los mismos; de modo que este ciclo es constante en su quehacer pedagógico.

En este mismo sentido, Elliot, J. (1994) señala: "Que la investigación acción aplicada a la educación tiene que ver con los problemas prácticos cotidianos, experimentado por los docentes más que problemas teóricos definidos por investigadores dentro de un área del conocimiento". 
Bio - grafía. Escritos sobre la Biología y su Enseñanza. ISSN 2027-1034

Edición Extraordinaria. p.p. 1415-1422

Memorias del IX Encuentro Nacional de Experiencias en Enseñanza de la Biología y la

Educación Ambiental. IV Congreso Nacional de Investigación en Enseñanza de la Biología.

\section{RESULTADOS}

En consecuencia, la dificultad principal de los estudiantes está relacionada con los niveles de observación e indagación que se ha evidenciado; lo que permite plantear desde el área de tecnología actividades que promueven el fortalecimiento de estas competencias, mediante un ciclo de acción reflexión enmarcado dentro de la investigación acción. Por otra parte, el docente debe planear sus sesiones de clases teniendo en cuenta el conocimientos profesional del profesor de ciencias teniendo en cuenta el equilibrio entre sus componentes como lo son "el conocimiento pedagógico, disciplinar, didáctico y del contexto" (Furió, 2014).

Entre tanto, implementar El Marco de la Enseñanza para la Comprensión utilizando como estrategias las rutinas de pensamientos y sobre todo la rutina veo, pienso y me pregunto, ha sido fundamental en este proceso, pues el estudiante a medida que la realiza con distintos objetos, situaciones del aula o tópicos trabajados va perfeccionando la competencia científica de la observación al igual que utiliza las palabras para realizar preguntas, por lo tanto ya estas son pocas dentro del nivel sin categorías y el docente al registrar la información obtenida mediante la elaboración de estas rutinas puede darse cuenta de los avances o dificultades en el proceso de enseñanza aprendizaje; de esta manera entraría a replantear nuevamente la estrategia para continuar con el curso de su investigación.

Teniendo en cuenta lo anterior se han encontrado los siguientes hallazgos: Clases más dinámicas y activas donde los estudiantes expresan literalmente "esta clase si me gustó", transformación en las prácticas pedagógicas del docente, estudiantes motivados frente a los tópicos generativos trabajados en clases, desarrollo del pensamiento científico y tecnológico de los estudiantes y por ende su capacidad de observación e indagación y clases mejor planeadas frente a las necesidades de los estudiantes.

Mediante la implementación de la rutina de pensamiento "Veo, Pienso, Me Pregunto", los estudiantes han desarrollado actividades de observación de láminas, objetos de su entorno, temáticas estudiadas, etc. Con base en la recolección y sistematización de la información correspondiente a esta estrategia, se ha podido determinar los resultados que prevalecen en cuanto a la Observación e Indagación que realizan los estudiantes (Ver gráfica 4).

\section{NIVELES DE OBSERVACIÓN}

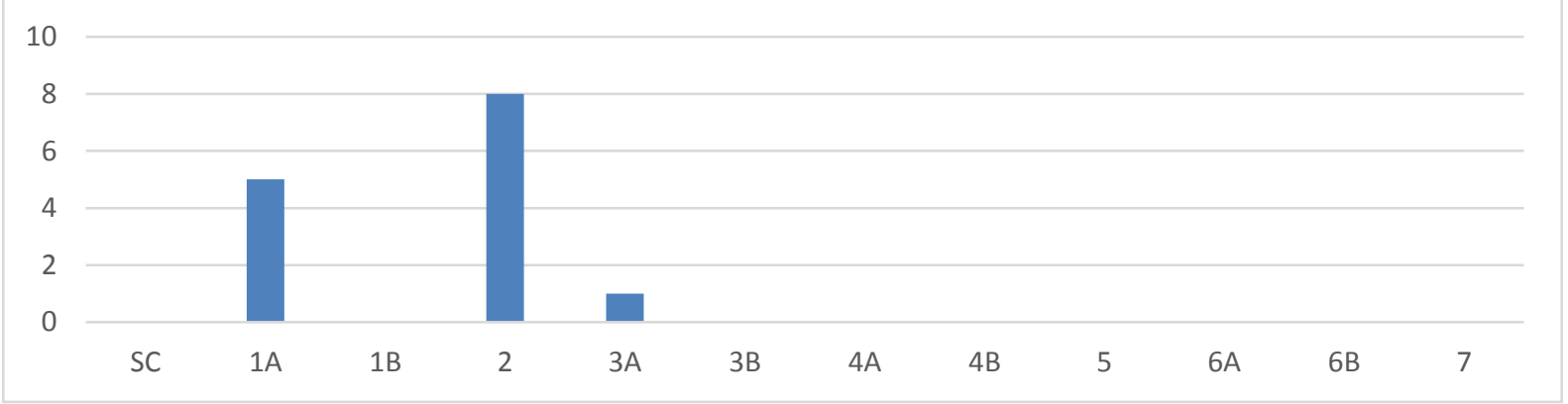

Gráfica 4. Consolidado de prevalencia de los niveles de observación realizadas por los estudiantes de Quinto 
Bio - grafía. Escritos sobre la Biología y su Enseñanza. ISSN 2027-1034

Edición Extraordinaria. p.p. 1415-1422

Memorias del IX Encuentro Nacional de Experiencias en Enseñanza de la Biología y la Educación Ambiental. IV Congreso Nacional de Investigación en Enseñanza de la Biología.

Además, en relación a las observaciones que los estudiantes van realizando la docente va tabulando la información referente a los niveles de indagación y tipología de preguntas usadas. (Ver resultados gráfica 5 ).

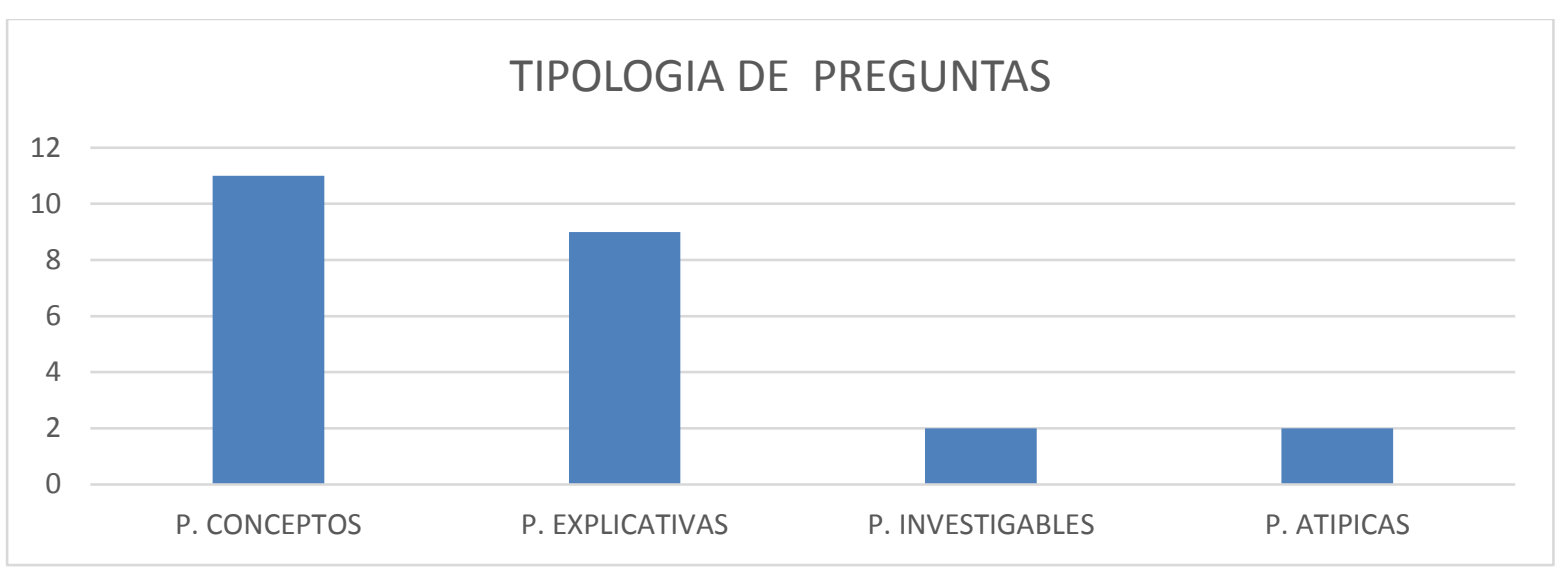

Gráfica 5. Resultados de la prevalencia en los tipos de preguntas planteadas por los estudiantes de Quinto.

Por otro lado, a partir de los resultados de los niveles de indagación la docente se permite ir guardando los resultados de las tipologías de preguntas que usan los estudiantes de Quinto al realizarse preguntas de lo observado. (Ver gráfica 6).

\section{CATEGORIAS DE LAS PREGUNTAS}

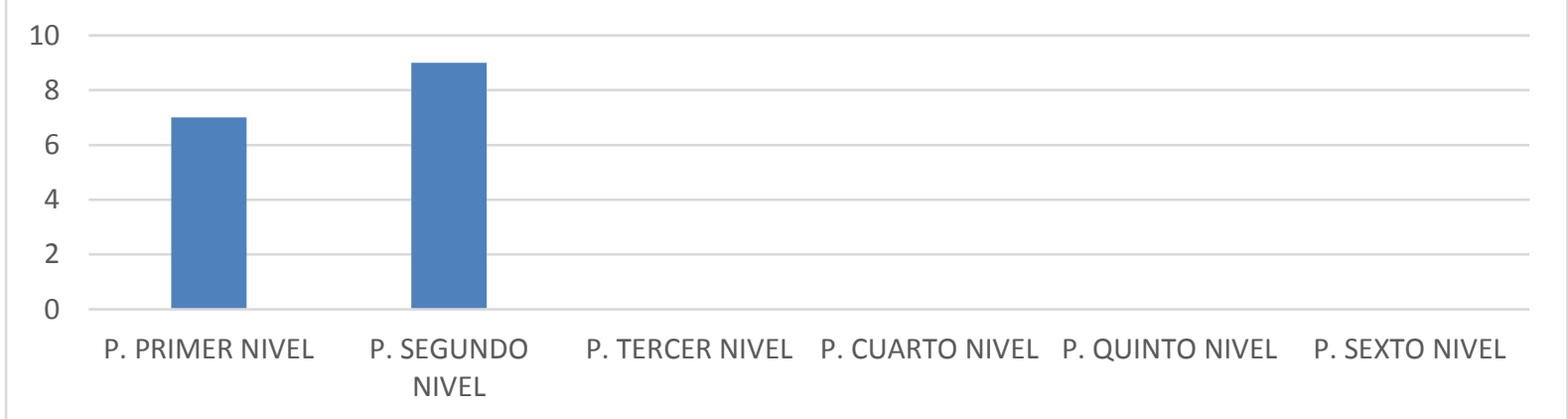

Gráfica 6. Resultados de prevalencia de los niveles de indagación en los estudiantes de Quinto.

\section{CONCLUSIONES}

El análisis de la problemática evidenciada en pruebas Saber, permitió realizar una reflexión en relación con el rol que el docente tiene en el desarrollo del pensamiento científico-tecnológico, específicamente en las competencias de observación e indagación de sus estudiantes; y cómo la transformación de las prácticas pedagógicas del docente 
Bio - grafía. Escritos sobre la Biología y su Enseñanza. ISSN 2027-1034

Edición Extraordinaria. p.p. 1415-1422

Memorias del IX Encuentro Nacional de Experiencias en Enseñanza de la Biología y la Educación Ambiental. IV Congreso Nacional de Investigación en Enseñanza de la Biología.

pueden favorecer la superación de las deficiencias presentes, por lo tanto, se pudieron tomar acciones que han posibilitado la mejoría de estas competencias científicas.

Entre tanto, uno de este ejercicio ha sido planear desde el marco de la EpC; teniendo en cuenta los componentes del conocimiento profesional del profesor de ciencias en cuanto a la didáctica, la pedagogía, la disciplina y el contexto. En consecuencia, el docente desde su quehacer pedagógico puede mejorar los niveles de comprensión de sus estudiantes, desarrollando las competencias científicas tecnológicas desde su área, y comprenda que es fundamental analizar, sistematizar e implementar reflexiones relacionadas con los conocimientos profesionales del profesor, al momento de planear una sesión de clases para lograr que el estudiante se apropie de aprendizajes significativos.

De la misma manera, los estudiantes han logrado superar las deficiencias en las competencias científicas de observación, formulación de preguntas e indagación; lo que le ha permitido obtener mejores resultados académicos por lo que han desarrollado comprensión en cada una de las áreas del conocimiento.

\section{REFERENCIAS BIBLIOGRÁFICAS}

Blythe, T. (2005). Enseñanza para la Comprensión: Cerrando la brecha entre teoría y la Acción. Magisterio, Vol. 14, pp.12.

Elliot, J. (2000). Investigación Acción Educativa. Morata. Recuperado de: http://www.terras.edu.ar/biblioteca/37/37ELLIOT-Jhon-Cap-1-y-5.pdf

Feldman, D. (2010). Didáctica General. Buenos Aires. Recuperado de: http://cedoc.infd.edu.ar/upload/Didactica_general.pdf

Furió, C. (9 de septiembre de 2014). Carles Furió: Investigando el Conocimiento Profesional del Profesor de Ciencias. [Archivo de video] Recuperado de: https://www.youtube.com/watch?v=JSd9yPIZYoc

Furman, M., \& García, S. (2014). Categorización de preguntas formuladas antes y después de la enseñanza por indagación. Praxis y saber, Vol. 5 (10), consultado en: 2 de julio de 2017. Recuperado de:

http://revistas.uptc.edu.co/index.php/praxis_saber/article/view/3023/2738

Meinardi, E. (2010). Educar en ciencias. Buenos Aires Argentina. Paidós

Richhart, R; Church, M; \& Morrinson, K. (2014). Hacer Visible el Pensamiento: Cómo promover el compromiso, la comprensión y la autonomía en los estudiantes. Buenos Aires Argentina. Paidós

Orientaciones Generales para la Educación en Tecnología. (2008). Bogotá. Recuperado de: http://www.mineducacion.gov.co/portal/men/Publicaciones/Guias/160915:Guia-No-30-Sercompetente-en-tecnologia-una-necesidad-para-el-desarrollo

Parga, D. (2015). El Conocimiento Didáctico del Contenido (CDC) en química. Bogotá Colombia. 
Bio - grafía. Escritos sobre la Biología y su Enseñanza. ISSN 2027-1034

Edición Extraordinaria. p.p. 1415-1422

Memorias del IX Encuentro Nacional de Experiencias en Enseñanza de la Biología y la

Educación Ambiental. IV Congreso Nacional de Investigación en Enseñanza de la Biología.

Santelices, L. (1989). Metodología de ciencias naturales para la enseñanza básica. Santiago de Chile: Andrés Bello 